\title{
O ESTILO DE VIDA DE PACIENTES HIPERTENSOS DE UM PROGRAMA DE EXERCÍCIO AERÓBIO: ESTUDO NA CIDADE DE TOLUCA, MÉXICOª
}

\author{
The Life Style of the Hypertensive Patient of a \\ Program of Aerobic Exercise: Study in the Toluca City, Mexico \\ El Estilo de Vida de Pacientes Hipertensos de un \\ Programa de Ejercicio Aerobio: Estudio en la Ciudad de Toluca, México
}

Maria Suely Nogueira²

\begin{abstract}
Resumo
A hipertensão arterial, assintomática e desconhecida pela maioria dos seus portadores, é importante fator de risco para complicações cardiovasculares e renais. Este é um estudo quantitativo e descritivo. Objetivou identificar os estilos de vida associados ao incremento do descontrole da pressão ar terial em pacientes hipertensos que acodem a um programa de exercício. A coleta dos dados ocorreu num centro de saúde do município do Toluca, México, durante agosto 2006 a março 2007. Foi utilizado um questionário aplicado a 200 pacientes hipertensos, através do referencial do "Modelo de Campo de Saúde da Lalonde". 0 estudo mostrou que o nível de pressão do grupo estudado possui relação estatisticamente significativa com o tabagismo, consumo de álcool e tratamento. A falta do controle da pressão arterial pode propiciar a evolução de doenças cardiovasculares e coronárias.
\end{abstract}

Palavras-chave: Estilo de vida. Hipertensão. Pressão Arterial. Enfermagem.

\begin{abstract}
Hypertension is an asymptomatic and an unnoticed disease for the majority of hypertensive individuals and an important long-term factor of risk for cardiovascular and renal complications. It is quantitative and descriptive study. The study aims to evaluate hypertenses's lifestyles in a program of aerobic exercise. The collection of data was made in a health center of Mexico City, during the months of August in 2006 to March in 2007. A questionnaire was applied to 200 hypertenses, using the referencial framework of Lalonde: "Field of Health Model". The study showed the existing statistic relationship between the levels of arterial pressure, smoking habits, consumption of alcohol and the monitoring of their blood pressure condition. The lack of control of the arterial pressure may propitiate the evolution of coronary and cardiovascular illnesses.
\end{abstract}

\section{Resumen}

La hipertensión arterial es asintomatica y desconocida por la mayorías de sus portadores y es un importante factor de riesgo para complicaciones cardiovasculares y renales. Este es un estudio cuantitativo y descriptivo. Con el objetivo de evaluar el estilo de vida de pacientes hipertensos registrados en un programa de ejercicio aerobio. La colecta de los datos ocurrió en un centro de salud del municipio de Toluca, México, durante agosto de 2006 a marzo de 2007. Fue utilizado un cuestionario aplicado a 200 pacientes hipertensos, a través del referencial del "Modelo Campo de Salud de Lalonde". El estudio muestra que existe relación estadística entre los niveles de presión arterial, con el tabaquismo, consumo de alcohol, y el tratamiento. La falta del control de la presión arterial puede propiciar la evolución de enfermedades cardiovasculares y coronarias.
Keywords:

Lifestyle. Hypertension. Blood Pressure. Nursing.
Palabras clave:

Estilo de Vida. Hipertensión. Presión arterial. Enfermería. 


\section{INTRODUÇÃO}

A hipertensão arterial sistêmica (HAS) é um importante fator de risco para o desenvolvimento de cardiopatia isquêmica e acidente vascular cerebral. Mundialmente, a HAS é doença de alta prevalência, e estima-se que existam 691 milhões de pessoas hipertensas, das quais 420 milhões vivem em países em desenvolvimento. Calcula-se que 15 milhões de hipertensos morram a cada ano; a Organização Mundial da Saúde (OMS) ${ }^{1}$ revelou que 0 acidente vascular cerebral (AVC) foi responsável por $30 \%$ dos óbitos ocorridos no mundo. Os resultados assinalam que essa doença está aumentando e as autoridades de saúde pública enfrentam atualmente um dos maiores desafios para o controle da pressão arterial e outras doenças crônicas ?

Pelas estimativas no México, a ocorrência da HAS apresenta valores que flutuam entre $10,2 \%$ e $30,5 \%$, em função dos critérios de medida utilizados ${ }^{3}$. A doença aumenta na faixa etária de 50 anos e mais, isto é, um em cada dois mexicanos é portador da HAS, segundo a Enquête Nacional de Saúde 4 .

Nas últimas décadas, as mudanças no panorama epidemiológico da população mexicana relacionadas com a diminuição das doenças infecciosas, combinadas com outras mudanças no estilo de vida, deram lugar ao aumento progressivo das doenças crônico-degenerativas e constituem a primeira causa de morte. Considera-se a HAS como um problema de saúde pública, já que favorece o surgimento de outras doenças graves, que comprometem órgãos nobres, conhecidos por "órgãos alvo", como o coração, encéfalo, rins, retina e vasos ${ }^{3,5}$.

Diante da associação entre HAS e o estilo de vida como fator de risco para o incremento do índice de morbidade e mortalidade por infarto agudo do miocárdio e DCV, optou-se por desenvolver este estudo utilizando como referencial teórico o Modelo de Campo de Saúde de Lalonde. Nos últimos 25 anos, ficou evidente que as principais causas de enfermidade $\mathrm{e}$ morte estavam relacionadas a três componentes: a biologia humana, o meio ambiente e o estilo de vida ${ }^{6}$.

Assim, observou-se que este último componente está fortemente associado aos hábitos de vida das pessoas e ao desenvolvimento da HAS; os mais discutidos são o sedentarismo, a obesidade, tabagismo e ingestão de bebidas alcoólicas. Por si só a doença já constitui um importante fator de risco para acometimentos cardiovasculares e coronarianos ${ }^{7}$.

Desta forma, o estilo de vida propicia o desenvolvimento e/ ou manutenção de fatores que comprovadamente afetam a própria saúde do indivíduo, incrementam o nível de doença ou morte prematura e elevam o índice de mortalidade pela doença ${ }^{6}$.

Como a doença é assintomática, o controle dos fatores de risco modificáveis é possível de ser realizado de forma relativamente simples, baseada na magnitude e na gravidade de suas manifestações ${ }^{8}$. Os pacientes portadores de hipertensão arterial devem ser orientados quanto à manutenção ou mudança de estilo de vida adequado e promoção do exercício, perda de peso, restringindo o consumo de sódio, álcool, cigarro, para a promoção de sua saúde e um melhor controle de sua $\mathrm{PA}^{9}$.

Dessa forma, o estudo teve como objetivo: avaliar o estilo de vida de pacientes hipertensos cadastrados em um programa de exercício aeróbio na cidade do Toluca, México.

\section{MATERIAIS E MÉTODOS}

0 estudo foi descritivo, de natureza quantitativa, realizado em um centro da saúde cidade de Toluca, México. Os dados foram coletados durante as atividades de exercício dos pacientes. A amostra foi constituída de 200 hipertensos cadastrados no programa de exercício aeróbio para portadores de doenças crônico-degenerativas. Os critérios para elegibilidade dos indivíduos nesta investigação foram: ter diagnóstico confirmado de HAS pelo médico do centro de saúde; participar de forma regular do programa de exercício; ser homem ou mulher acima de 18 anos, e ter desejo voluntário de participar no estudo, por meio da assinatura do termo de consentimento livre e esclarecido.

Foi assegurado aos participantes sigilo quanto às informações prestadas. A pesquisa foi aprovada pelo Comitê de Bioética em Investigação da Escola de Enfermagem de Celaya da Universidade de Guanajuato, México, conforme o disposto no artigo 14, inciso VII (Ley General de Salud en Matéria de Investigación), sendo obtido o consentimento livre e esclarecido, que foi assinado pelos participantes ${ }^{10}$.

0 instrumento para coleta de dados constou de um questionário aplicado oralmente com perguntas sobre os quatro conceitos de Lalonde?. A coleta dos dados foi realizada durante os meses de agosto de 2006 a março de 2007, no horário da manhã, do programa de exercício. Por meio de entrevista individual, obtiveram-se os dados dos pacientes, tendo sido informados aos participantes os dados antropométricos (peso, altura, circunferência abdominal e quadril) e de valores da pressão arterial.

A pressão arterial foi aferida, utilizando-se esfigmomanômetros do tipo aneróide e estetoscópios, previamente testados e devidamente calibrados, e manguito com largura de bolsa de borracha compatível com a circunferência braquial do indivíduo, de acordo com as recomendações da Norma Oficial Mexicana (2006) ${ }^{11}$ e V Diretrizes Brasileiras de Hipertensão Arterial (2006) ${ }^{12}$.

Consideraram-se pressão arterial sistólica a primeira fase de Korotkoff e a quinta fase (último ruído) da diastólica. Estabeleceram-se valores para HAS com cifras iguais ou maiores a 140/90 mmHg, seguindo a Norma Oficial Mexicana (2006) ${ }^{10}$. Ressalta-se que, para este estudo, consideraram-se hipertensos os indivíduos com diagnóstico prévio de HAS que recebiam medicação anti-hipertensiva. Conseqüentemente pôde-se verificar em que nível de controle se encontravam os pacientes portadores da hipertensão arterial ${ }^{8}$.

A aferição da pressão ar terial foi realizada ao final da entrevista, momento em que o paciente permaneceu comodamente sentado em repouso de 5 a 10 minutos. Recomendou-se, também, não cruzar as pernas, ter a bexiga urinária vazia, além de não ter se alimentado, não ter ingerido bebida alcoólica e nem feito exerćício físico nos trinta minutos anteriores à aferição. Os registros foram feitos em milímetros de mercúrio.

Depois de serem coletados, os dados foram codificados numa base de programa estatístico (SPSS, versão 11, em espanhol) que permitiu as medidas de freqüência e 
percentagens. Recorreu-se à estatística descritiva, isto é, a medidas de tendência central (média), de dispersão (desviopadrão) e percentagens. Análise de associação de variáveis: odd ratio $(0 R)$, regressão linear.

\section{RESULTADOS E DISCUSSÃO}

Dos 200 pesquisados, encontrou-se que 91 (45,5\%) têm pressão controlada e 109 (55,5\%), não controlada, com média e desvio-padrão de PAS 134,6士16,16 (mmHg). Deles, 161 $(80,5 \%)$ são do sexo feminino e 39 (19,5\%), do masculino, com idade maior de 18 anos, com média de idade de (56士14) anos. Confrontando com outra pesquisa ${ }^{13}$, observa-se certa similaridade, talvez em virtude de sua disponibilidade para comparecer ao programa de exercício e também da maior longevidade das mulheres em relação aos homens.

Na Tabela 1 estão apresentados os resultados obtidos junto a essa população mexicana, que mostram o predomínio da faixa do indivíduo acima de 60 anos e do sexo feminino; 0 processo de envelhecimento envolve um conjunto de fatores que propicia os agravos à saúde com o passar dos anos, tais como alterações funcionais, tornando fator predisponente para doenças do sistema cardiovascular e coronarianas ${ }^{12,13}$.

Tabela 1: Características sociodemográficas dos pacientes hipertensos. Toluca, México, 2007 ( $n=200)$.

\begin{tabular}{lcc}
\hline VARIÁVEIS & INDICADORES & NÚMERO \\
\hline Idade & \% \\
Sexo & & $56 \pm 14$ \\
& Feminino & $161(80,5)$ \\
Estado Civil & Masculino & $39(19,5)$ \\
& Casado & $118(59)$ \\
& Viúvo & $50(25)$ \\
Ocupação & Solteiro & $32(16)$ \\
& Do Lar & $148(74)$ \\
& Comerciante & $29(14,5)$ \\
& Aposentado & $8(4)$ \\
Escolaridade & Professor & $15(7,5)$ \\
& Baixa & $157(78,5)$ \\
& Média & $22(11)$ \\
Renda Mensal & Alta & $21(10,5)$ \\
& $\leqslant 350$ Reais & $145(72,5)$ \\
& $\geqslant 350$ Reais & $55(28)$ \\
\hline
\end{tabular}

Pode-se observar, em relação ao estado civil, que 118 (59\%) hipertensos eram casados; 50 (25\%), viúvos; e 32 (16\%), solteiros, separados ou divorciados. No tocante à ocupação, 148 (74\%) eram do lar; 29 (14,5\%), comerciantes; 15 (7,5\%), professores; e apenas 8 (4\%) eram aposentados. Em relação ao grau de escolaridade, 157 (78,5\%) hipertensos apresentavam baixo nível de escolaridade (menos de cinco anos); 21 (11,5\%), nível médio (entre seis a oito anos); e 21 (10,5\%) nível alto (mais de 12 anos de escolaridade). Observa-se que existe um predomínio de analfabetos funcionais, que não conseguem ler e escrever inteiramente, de modo que a interação entre educação e saúde de certa forma pode inferir diretamente na compreensão das orientações dos profissionais de enfermagem ${ }^{13,14}$.
Quanto à renda, 145 (72,5\%) indicaram receber menos de dois salários mínimos/mês, alguns sobrevivem com apenas dois dólares ao dia, o equivalente a quatro reais/dia, e 55 (28\%) recebem mais de 350 reais mensais. Esses dados mostram que os hipertensos com menos de cinco anos de escolaridade têm menos oportunidade de emprego e que, por conseguinte, o estado financeiro interfere no tratamento e nas mudanças do estilo da vida necessárias, pois o futuro se mostra incerto e desconcertante para o controle da hipertensão ${ }^{15,16}$.

Tabela 2: Relação do estilo de vida com o controle da pressão arterial do paciente hipertenso. Toluca, México, 2007 ( $n=200)$.

\begin{tabular}{|c|c|c|c|}
\hline VARIÁVEIS & $\begin{array}{c}\text { CONTROLE DA } \\
\text { PRESSÃO } \\
91(45,5 \%)\end{array}$ & $\begin{array}{c}\text { DESCONTROLE DA } \\
\text { PRESSÃO } \\
109(54,5 \%)\end{array}$ & $p^{*}$ \\
\hline Tempo da doença & & & 0,325 \\
\hline$\leqslant 5$ anos & $46(23)$ & $65(32,5)$ & \\
\hline$\geqslant 5$ anos & $45(22,5)$ & $44(22)$ & \\
\hline Tratamento & & & 0,048 \\
\hline Seguimento & $90(45)$ & $97(48,5)$ & \\
\hline Abandono & $1(0,5)$ & $12(6)$ & \\
\hline História familiar & & & 0,806 \\
\hline Positiva & $43(21,5)$ & $50(25)$ & \\
\hline Negativa & $48(24)$ & $59(29,5)$ & \\
\hline Atividade física & & & 0,129 \\
\hline Positiva & $66(33)$ & $68(34)$ & \\
\hline Negativa & $25(12,5)$ & $41(20,59)$ & \\
\hline Diabetes & & & 0,007 \\
\hline Positivo & $15(7,5)$ & $5(2,5)$ & \\
\hline Negativo & $76(38)$ & $104(52)$ & \\
\hline Tabagismo & & & 0,026 \\
\hline Positivo & $27(13,5)$ & $18(9)$ & \\
\hline Negativo & $64(32)$ & $91(46,5)$ & \\
\hline Consumo de álcool & & & 0,069 \\
\hline Positivo & $26(13)$ & $33(16,5)$ & \\
\hline Negativo & $65(32,5)$ & $76(35,5)$ & \\
\hline Estresse & & & 0,88 \\
\hline Positivo & $75(37,5)$ & $99(49,5)$ & \\
\hline Negativo & $16(8)$ & $20(10)$ & \\
\hline IMC & & & 0,477 \\
\hline Normal & 16(8) & $17(8,5)$ & \\
\hline Pré-obesidade & $43(21,5)$ & $49(24,5)$ & \\
\hline Obesidade (I,II,III) & $32(16)$ & $43(21,5)$ & \\
\hline
\end{tabular}

* quiquadrada

A Tabela 2 contém dados referentes ao tempo da doença: 111 (45,5\%) pacientes têm a doença a menos de cinco anos e $99(49,5 \%)$ têm a doença há mais de cinco anos. Esse dado não tem associação estatisticamente significativa $(p<0,325)$ com o controle da pressão arterial. Desses pacientes, $90(45 \%)$ afirmaram seguir tratamento sistemático, os quais apresentavam PA controlada, e 97 (48,5\%), embora afirmassem fazer tratamento, ainda não estavam com a PA controlada. Os dados também mostram que 13 (6,5\%) pacientes afirmam abandonar o tratamento, o que interfere no controle da pressão arterial. Esses dados têm associação estatisticamente significativa $(p<0,048)$ com 0 controle da pressão arterial e com a correlação linear. A chance de aumentar a PA foi de 11,34 vezes maior naqueles que abandonaram 0 
tratamento em relação àqueles que seguiam o tratamento, enquanto o intervalo de confiança ficou entre 1,46 e 87,36 (Tabela 3).

Tabela 3: Razão de risco para as variáveis significativas na análise do paciente hipertenso. Toluca, México, 2007 ( $n=200)$.

\begin{tabular}{|c|c|c|c|}
\hline & RISCO & RELATIVO & $\begin{array}{cc}\text { INTERVALO } & \text { DE } \\
\text { CONFIANÇA } & 95 \%\end{array}$ \\
\hline Tratamento & & 11,34 & $1,46-87,36$ \\
\hline Tabagismo & & 2,13 & $1,084-4,196$ \\
\hline Álcool & & 1,038 & $0,790-1,364$ \\
\hline Estresse & & 1,053 & $0,510-2,176$ \\
\hline
\end{tabular}

Nos pacientes de diagnóstico recente (com menos de cinco anos) foi comum a inexistência de sintomas nos primeiros anos. Um fato importante é que o indivíduo portador da hipertensão arterial pode ter a pressão arterial alta e não saber; por esta razão, é chamada de "assassina silenciosa". Nesta etapa pode estar acompanhada por períodos de descontrole da pressão arterial; portanto, existe um desafio muito importante de manter o paciente com cifras tensionais controladas. Assim também os profissionais da saúde devem verificar a resposta ao tratamento, com vista a identificar se o tratamento está sendo bem tolerado pelo paciente $e^{14,17}$.

0 tratamento da hipertensão é um grande desafio para a equipe multidisciplinar, especialmente em relação à adesão ao tratamento. Estudos realizados no Brasil ${ }^{15,16}$ e no México 2,3 têm demonstrado que a maioria dos hipertensos tem dificuldade de adesão ao tratamento. Na prática clínica observa-se que os pacientes retornam às consultas médicas de forma regular devido ao descontrole da pressão arterial, e, em grande parte, as mortes que ocorrem cada ano são conseqüência direta da hipertensão arterial ou de suas complicações sobre o sistema cardiovascular ${ }^{13,16}$.

Em relação à história familiar, pôde-se perceber, na Tabela 2, que 93 (46,5\%) hipertensos tinham antecedentes familiares positivos, pelo menos um familiar com hipertensão arterial, diabetes mellitus, obesidade e doença cardiovascular, enquanto 107 (53,5\%) apresentavam história familiar negativa para DCV, e esse dado não tem associação estatisticamente significativa $(p<0,806)$ com o controle da pressão arterial. Outros estudos ${ }^{18}$ demonstraram que a história familiar positiva é fator de risco para desenvolver acidente vascular cerebral ou problemas cardíacos; os hipertensos relataram ter parentes consangüíneos diretos (pai, mãe, irmãos e avós) com as doenças. A HAS possui um componente hereditário, sendo fator de risco para doenças como diabetes mellitus e infarto agudo do miocárdio ${ }^{8,12}$.

0 estilo de vida, proposto pelo modelo de campo de saúde, inclui decisões tomadas pelo indivíduo e que afetam sua própria saúde. Para o autor, os efeitos dos riscos modificáveis relacionados com as doenças cardiovasculares, um problema da modernidade, refletem-se nos hábitos apresentados nos estilos de vida ${ }^{13,17}$.

Em relação ao estilo de vida relacionado à atividade física realizada pelos hipertensos, pode-se verificar que 134 (77\%) hipertensos realizavam algum tipo de atividade física, e 66 (33\%) não realizavam nenhuma atividade. Esse dado não tem associação estatisticamente significativa $(p<0,129)$ com o controle da pressão arterial.

A prática de alguma atividade física é decisão tomada pelo indivíduo como consciência dos efeitos de riscos para melhorar sua própria saúde. Para este autor, os efeitos dos riscos autocriados relacionados com as doenças cardiovasculares refletem-se nos hábitos presentes nos estilos de vida ${ }^{13,18}$.

Diversos estudos sobre o indivíduo que não realiza exercício mostram aumento da PA como fator de risco para as doenças do coração e do cérebro. Múltiplos estudos assinalam que um paciente submetido a um programa de exercício controlado pode reduzir a PA e aumentar a capacidade cardiovascular; pode-se também ter, em alguns casos, uma forma única de tratamento não farmacológico para o controle da hipertensão, em outros basta reduzir a dose dos medicamentos ${ }^{19}$. Portanto, um programa de exercício aeróbio ajuda a fortalecer o coração e o sistema osteomuscular, a baixar o peso, além de contribuir no controle da PA e prevenir os efeitos secundários das doenças crônicas como dislipidemia, obesidade e o diabetes mellitus, resultando em melhor qualidade de vida com custo baixo e risco mínimo. Não se sabe exatamente qual é o mecanismo envolvido para a queda da PA. 0 exercício deve ser realizado de acordo com a idade de cada pessoa, e fazer caminhada suave por um período de meia hora a 45 minutos dois ou três dias por semana é suficiente para a maior parte dos hipertensos 16 .

Quanto ao diabetes mellitus, $20(10 \%)$ hipertensos eram diabéticos, deles, somente $15(7,5 \%)$ estavam com pressão arterial controlada, e 180 (90\%) afirmavam não ser diabéticos; esse dado apresentou associação estatisticamente significativa $(p<0,007)$ com o controle da pressão arterial; neste sentido, as doenças crônicas são responsáveis pelas principais causas de morte entre os hipertensos ${ }^{13,18}$.

Sobre o tabagismo, 45 (14,58\%) hipertensos eram fumantes e consumiam 10 cigarros ao dia; a causa principal para o uso de cigarro foi o estresse, seguida de situações conflitantes no trabalho. Esse dado tem associação estatisticamente significativa $(p<0,026)$ com o controle da pressão arterial. Encontrou-se associação estatística com a HAS e tabagismo. A Tabela 3 mostra que OR é a chance 2,13 vezes maior de aumentar a PA, com um intervalo de confiança de 1,084 - 4, 196, mostrando que aqueles fumantes tinham maior chance de aumentar a PA do que aqueles que não eram fumantes. É considerado pelo Modelo de Campo de Saúde de Lalonde como o mais importante fator de risco evitável de morbidade e de mortalidade ${ }^{13}$. Apesar das evidências científicas que apontam para os malefícios do tabagismo, sua alta prevalência faz inúmeras vítimas para doenças cardiovasculares e ateroescleróticas ${ }^{13,15,18}$.

Nessa população de hipertensos, 59 (29,5\%) pacientes indicaram consumir bebida alcoólica, sendo a tequila a bebida mais consumida, seguida da cerveja. Esse dado apresenta associação estatisticamente significativa $(p<0,069)$ para 0 controle da pressão arterial. Os dados demonstraram que tanto o tabagismo como o etilismo têm mais chance de apresentar formas malignas da doença hipertensiva, que eleva a incidência 
de morte súbita ${ }^{13}$. Outros estudos observaram que a restrição da ingestão de álcool é uma medida eficaz na redução da $\mathrm{PA}^{15}$.

Quanto ao estresse, ao serem questionados, 164 $(82 \%)$ hipertensos indicaram que se consideravam pessoas nervosas ou estressadas. As situações que mais lhes provocavam estresse eram variadas, tais como: situações familiares e do lar, atividades no trabalho e aquelas ligadas aos problemas sociais, como falta de dinheiro, baixos salários e dificuldades no dia-a-dia ${ }^{13,20}$.

Por outro lado, pesquisou-se com que freqüência o estresse ocorria entre eles, e 87 (53\%) pacientes indicaram que se sentiam estressados durante os sete dias da semana, $25(15,2 \%)$ indicaram que o estresse ocorria uma vez por semana e $52(31 \%)$ indicaram que o estresse ocorria de duas a seis vezes por semana. Durante as entrevistas, questionou-se o que utilizavam para relaxar e diminuir 0 estresse, e 99 (49\%) indicaram que não faziam nada, 19 $(9,5 \%)$ procuravam a meditação, respiração e exercício, e 46 (23\%) optavam por leitura, assistir televisão, deitar-se e escutar música ou andar pelas ruas. Esses dados não apresentam associação estatisticamente significativa $(p<0,88)$ com controle da pressão arterial. A chance de aumentar a pressão arterial foi de OR 1,053 vezes maior, com intervalo de confiança entre 0,510 - 2,176 (Tabela 3). As mulheres que indicavam ser nervosas apresentavam maior chance de aumento da pressão arterial do que aquelas que não referiam ser ansiosas ou nervosas. Estudos sobre os aspectos emocionais evidenciam que a dificuldade de expressar os sentimentos hostis comprometem a saúde, como no caso da HAS e suas complicações ${ }^{13,20}$.

Estresse e emoções crônicas aumentam os batimentos e esforços cardíacos e a pressão sangüínea. Pessoas com esta característica normalmente são tidas como sujeitos com personalidade tipo A, por serem ambiciosos e terem desejos persistentes de progredir e necessidade de reconhecimento; têm ocupações múltiplas, são responsáveis, hostis e irritáveis, com sentido de urgência ante as tarefas, impacientes, com hábitos motores inquietos e respostas verbais entrecortadas, preocupam-se em se destacar em qualquer atividade que exerçam. Essa personalidade gera estresse crônico que afeta o músculo cardíaco e os corpos sangüíneos e favorece a formação de placas arteroescleróticas ${ }^{20}$.

Quanto ao IMC, 16 (8\%) encontram-se normais, com pressão controlada, já 17 (8,5\%) não possuem a pressão arterial controlada, ainda que tenham IMC normal; préobesidade: 43 (21,5\%) têm pressão controlada e 49 (24,5\%) não estão com a pressão controlada, e 32 (16\%) têm obesidade entre o nível I e o nível III. Quanto à RCC, a maioria desses hipertensos apresenta risco de nível III, 43 (21,5\%). Esses resultados indicam mais uma vez o sucesso do controle da pressão arterial para milhões de pacientes, através de modificações nos estilos de vida, da redução de peso, devido ao papel previsível da obesidade para o agravamento da hipertensão; ao reduzir peso também se consegue reduzir os requerimentos na dose de fármacos anti-hipertensivos $5,14,15$.

\section{CONSIDERAÇÕES FINAIS}

Os resultados deste estudo revelam que os estilos de vida dos pacientes portadores de hipertensão arterial, de acordo com o Modelo Campo de Saúde de Lalonde, apresentam fatores de risco relacionados com o controle da pressão arterial e existe a predisposição das doenças cardiovasculares, refletindo-se nos hábitos presentes no estilo de vida desses hipertensos.

Constatou-se que o grupo estudado apresenta fatores de risco, tais como tabagismo, consumo de álcool, sedentarismo, não seguimento do tratamento e estresse, aumentando, assim, o risco de descontrole da pressão arterial e das complicações com outras doenças cardiovasculares. É importante entender que os estilos de vida dos sujeitos estão relacionados com o controle da HAS. Desse modo, existe relação entre o cuidado da saúde e o estilo de vida.

Também, a mudança de comportamento depende, em parte, da vontade dos hipertensos pesquisados para decidir se querem mudar, e o que precisam mudar. Por outro lado, os profissionais de emfermagem têm a responsabilidade no fornecimento de cuidados de saúde primários, conscientizados sobre uma realidade nas ações do dia-adia para que o paciente portador da hipertensão arterial tome as melhores decisões que permitam maior controle da pressão arterial e prevenção de outras doenças.

No que se refere ao seguimento do tratamento terapêutico anti-hipertensivo para essa população pesquisada, parece tarefa impossível para alguns deles, pois, muitas vezes podem estar dispostos a seguir o plano terapêutico, mas existem condições que impedem a adesão, que, nesta pesquisa, foram as condições econômicas, baixo nível de escolaridade e a renda familiar mensal. Os hipertensos vivem com menos de dois dólares ao dia, e muitas vezes não é possível satisfazer as necessidades básicas como a alimentação; portanto, essas e outras condições não garantem a adesão ao tratamento.

Os resultados deste estudo contribuíram de forma significativa para um olhar mais reflexivo a respeito de nossa prática profissional, através de intervenções de enfermagem, visando à prevenção e/o controle dos fatores de risco modificáveis.

\section{Referências}

1. Organização Mundial da Saúde-OMS. Comunicado de Imprensa. 2000 [on-line] [citado abr 2007]. Disponível em: www.whoafr.org/ press/portuese/2000/regionalcommitte/rc501006.html>.

2. Rosas PM. La hipertensión arterial en México y su relación con otros factores de riesgo. Arch Cardiol México 2003; 73(6): 137- 40.

3. Mendoza GC. Guías del Instituto Nacional de Cardiología Ignacio Chávez para el tratamiento de la hipertensión arterial sistémica. Arch Cardiol México 2006; 76(2). 
4. Carrasco RR, Pérez CR, Oviedo MM, Morales RH. Análisis de una década de investigación en hipertensión arterial en México. Gac Méd, México 2004; 140(6): 611-18.

5. Velazquez MO, Rosas PM, Lara E A, et al. Hipertensión arterial en Mexico: resultados de la Encuesta Nacional de Salud, 2000. Arch Cardiol México 2002: 72-84.

6. Dever GEA. Epidemiologia y administración de los servicios de salud.OPS/OMS; 1991. p. 125-322.

7. Lalonde M. A new perspectiva on the health of canadians: a working document. Otawa (CA); 1974.

8. Simão M, Nogueira MS, Hayhashida M, Cesarino EJ. Doenças cardiovasculares: perfil de trabalhadores do sexo masculino de uma destiladora do interior paulista. Rev Eletr Enferm 2002; [citado 12 maio 2007]; 4(2): 27-35. Disponivel: <http://www.fen.ufg.br>.

9. Verdecchia P, Favio A. $7^{\circ}$ Joint National Committee on the prevention, detection, evaluation, and treatment of high blood pressure - JNC. Rev Cardiol 2003: 55(9).

10. Ley General de Salud en Materia de Investigación Para la Salud México. 12aed. México(MX): Porrúa; 2004. Comite de Bioética en Investigación de la Escuela de Enfermería de Celaya de la Universidad de Guanajuato, México. (conforme lo dispuesto en el artículo 14, incisoll).

11. Norma Oficial Mexicana. para la prevención, tratamiento y control de hipertensión arterial-NOM-043-SSA2-2005) 2000. [citado 12 março 2007] Disponível em: <http://www.salud.gob.mx>..

12. Sociedade Brasileira de Hipertensão. Sociedade Brasileira de Cardiologia. Sociedade Brasileira de Nefrología. V Diretrizes Brasileiras de Hipertensão Arterial. São Paulo(SP); 2006.

13. Teixeira ER, Lamas AR, Costa e Silva J, et al. 0 estilo de vida do cliente com hipertensão arterial e o cuidado com a saúde. Esc Anna Nery Rev Enferm 2006 dez; 10(3): 84.
14. Reiners OA. Interação profissional de saúde e usuário hipertenso: contribuição para não-adesão ao regime terapêutico. [tese de doutorado]. Ribeirão Preto(SP): Escola de Enfermagem /USP; 2005. 15. Stipp MDC, Leite JL, Cunha NM, Assis LS, Andrade MP, Simões RD. 0 consumo do álcool e as doenças cardiovasculares: uma análise sob o olhar da enfermagem. Esc Anna Nery Rev Enferm 2007 dez; 11(4): 581-85.

16. Araujo GBS, Garcia TR. Adesão ao tratamento anti-hipertensivo: uma análise conceitual. [on-line] jul 2006. Rev Eletr Enferm [acesso 15 nov 2006]. Disponível em: <http://www.fen.ufg.br>.

17. Dantas RAS, Colombo RCR, Aguilar OM. Perfil de mulheres com infarto agudo do miocárdio, segundo o modelo de "Campos de Saúde". Rev Latino-am Enfermagem 1999; 3(3): 63-8.

18. Siqueira CFP, Veiga VE, Moacir LDAJ. A hereditariedade como fator de risco para hipertensão arterial: algumas considerações. Rev Bras Enferm 2006; 2(6): 331-35.

19. Polito DM, Farrinatti PTV. Comportamento da pressão arterial após exercício contra-resistência: uma revisão sistemática sobre variáveis determinantes e possíveis mecanismos. Rev Bras Med Esporte 2006; 6(12): 25-35.

20. Chavez C, Cade VN. Efeitos da ansiedade sobre a pressão arterial em mulheres com hipertensão. Rev Latino-am Enfermagem 2004; 12(2): 162-67.

\section{Nota}

aEste trabalho é parte da tese de Doutorado apresentado à Escola de Enfermagem de Ribeirão Preto da Universidade de São Paulo, Brasil, decorrente de convênio firmado com a Faculdade de Enfermagem e Obstetrícia de Celaya da Universidade de Guanajuato, México. 